\title{
Should we be 3D printing patient specific implants and using them?
}

\author{
Mohamed Khalid* \\ Department of Orthopedics, Saudi Arabia

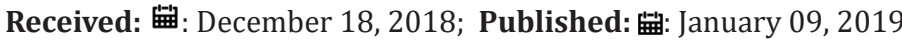 \\ *Corresponding author: Mohamed Khalid, Department of Orthopedics, College of Medicine, Saudi Arabia
}

\section{Opinion}

3D printing (3DP) builds objects layer by layer. It is widely believed that 3DP could prove to have as profound an impact on the manufacturing world as the PC and the Internet on the information world [1].

For

a) 3DP enables the design, and efficient manufacture, of personalized implants and could drive the transition from mass production to mass customization, in which each implant produced is customized to be exactly reciprocal to the size/ shape of the body part at a minimal production cost.

b) Implant designs, in the form of STL files, rather than implants, could move around the world to be printed anywhere by any printer that can meet the design parameters.

c) Distribution would shift from a 'push' to a 'pull' model with items made only on an as-needed basis, Hence, investments on inventory management are minimized.

d) It would be possible to vary the internal structure of an implant to match the part that it is re-enforcing. For example, long bones are dense in the middle and porous towards either ends. An implant being used to fix a fracture at the junction of the dense/porous part, could also have matching dense/porous sections. This would minimize the problem of modulus mismatch.

e) The cost of producing an implant by 3DP would be a fraction of the cost at which the implant is currently sold. For most of the established implant designs, their patents would have expired. Therefore, it should not be illegal to make them.

\section{Against}

a) Currently, there are no established standards for 3DP implants. Depending upon the type of printer as well as the raw materials the mechanical performance could vary substantially. Also, there could be a considerable batch to batch variation in mechanical performance.

b) If implant designs are moved around using the internet, there is potential for piracy and design theft. If implants printed from such pirated designs fail, it would be difficult/impossible to assign responsibility for the failure.

The solution to the above problems could be to print implants only at designated centers using high-end machines and to print identical implants in duplicate so that one of them could undergo standard mechanical testing for quality assurance. If there are limited number of printing centers, it would be easier to implement enhanced internet security protocols for the digital data. The established implant manufacturers would push back as they would lose a major share of the market. They would argue that they have made huge investments on R\&D to develop the current designs that are patent protected. Patients who opt for the 3DP implants would be deprived of these latest improvements.

With rare exceptions, most of the so-called enhanced design features are small incremental changes without convincing or evidence-based value, that have been introduced mainly for commercial reasons. With regard to the implants with expired patents, these companies have already made enormous profits, with markups as high as 100-200 times. In any case the option of using these regular implants could be given to the patients and they should be allowed to choose.

\section{The Intellectual Property Debate}

Copyrights and patents are the main instruments of IP protection. A copyright is designed to protect original creative work that is fixed in a tangible medium (ie. Sufficiently permanent or stable). Copyrights, conventionally, cover only aesthetic content 
and cannot be attached to producing functional parts. Therefore, they are not applicable to protecting digital files for 3DP of implants. Patents, on the other hand are designed to cover functional parts. If a 3D printer is used to reproduce a patented object, it is an infringement of the patent. However, infringement of a patented invention requires infringement of an entire invention. Novelty is an absolute primary requirement for the grant of a patent. A great majority of patented inventions, in reality, will consist of many existing inventions working together in a new way. Therefore, copying those non-novel parts of the patent would not constitute an infringement.

\section{Shaping the Future}

There is no doubt that 3DP is a disruptive technology and will have a profound influence on surgical implant technology. It is imperative, that as a respected and self-regulated profession we take responsibility and set the agenda, keeping our patient's interest foremost. By setting up our own regulatory bodies for 3DP, we can ensure that the current intellectual property laws are scrupulously followed and that a few bad apples do not provide an excuse to the implant industry to deny our patients the fruits of this technology. In spite of our best intentions it is possible that some people will infringe patent laws. In order to prove infringement, the patent owner should prove that the patented device was actually replicated and used. Unlike with copyright infringement the mere possession or downloading of the implant STL file is not

\section{ISSN: 2574-1241}

DOI: 10.26717/BJSTR.2019.12.002323

Mohamed Khalid. Biomed J Sci \& Tech Res

(C) (P) This work is licensed under Creative Commons Attribution 4.0 License

Submission Link: https://biomedres.us/submit-manuscript.php enough to prove infringement. However, should they somehow manage to prove it or even manage to create reasonable suspicion, patent owners might then resort to the doctrine of contributory infringement [2]. This might allow them to target 3D printer manufacturers and creators of digital content on the basis that they enabled the end users to infringe. If such a thing happened, it may potentially lead to the manufacturers and designers prohibiting the use of their machines and designs for medical implant production.

In conclusion, we should not forget that medical expenses are skyrocketing and implant costs contribute significantly to health care expenses. In the US, the leading cause for bankruptcy is medical illness [3]. It should be a part of our role as patient advocates to keep the interests of our patients above all else. We should endeavor to create awareness of the potential benefits of 3DP implants and ensure un-impeded access to this exciting technology, so that if and when the implant industry tries to influence the debate and introduce restrictive legislation, their claims of fighting pirates and scofflaws should fall on wise ears. We owe it to our patients.

\section{References}

1. T Campbell, C Williams, O Ivanova, B Garrett (2011) Could 3D printing change the world. Atlantic council, p. 16.

2. OB Roberts (1898) Contributory infringement of patent rights. Harvard Law Review 12: 35.

3. Himmelstein DU, Thorne D, Warren E, Woolhandler S (2009) Medical bankruptcy in the United States: Results of a national study. The American journal of medicine 122(8): 741-746.

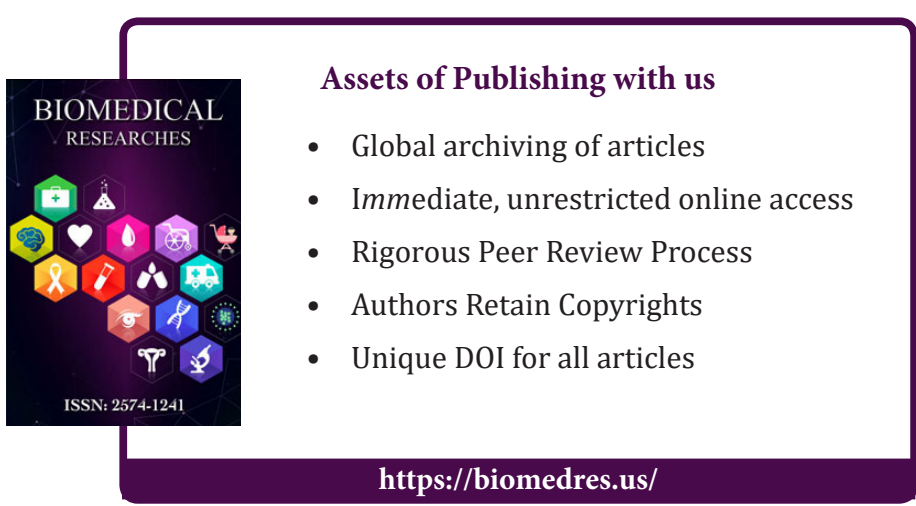

Cite this article: Mohamed Khalid. Should we be 3D printing patient specific implants and using them?. Biomed J Sci \& Tech Res 12 (5)-2019. 